\title{
Boosting Algal Bloom by Five-Fold with AIEgens: towards the Development of Biofactory
}

Haixiang Liu, Haotian Bai, Neng Yan, Tin Yan Wong, Dongfeng Dang, Jen-Shyang

Ni, Ryan T. K. Kwok*, Wen-Xiong Wang* and Ben Zhong Tang*

Dr. H. Liu, Dr. H. Bai, T. Y. Wong, Dr. D. Dang, Dr. J.-S. Ni, Dr. R. T. K. Kwok and

Prof. B. Z. Tang

Department of Chemical and Biological Engineering, Department of Chemistry, The Hong Kong Branch of Chinese National Engineering Research Center for Tissue Restoration and Reconstruction and Institute for Advanced Study, The Hong Kong University of Science and Technology (HKUST), Clear Water Bay, Kowloon, Hong Kong, China

E-mail: chryan@ust.hk; tangbenz@ust.hk

Dr. N. Yan and Prof. W-X. Wang

School of Energy and Environment, State Key Laboratory of Marine Pollution, City University of Hong Kong, Kowloon, Hong Kong, China

E-mail: wx.wang@cityu.edu.hk

Dr. R. T. K. Kwok and Prof. B. Z. Tang

HKUST-Shenzhen Research Institute, No. 9 Yuexing 1st RD, South Area, Hi-tech Park, Nanshan, Shenzhen 518057, China

Prof. B. Z. Tang

NSFC Center for Luminescence from Molecular Aggregate, Guangdong Innovative Research Team, SCUT-HKUST Joint Research Laboratory, State Key Laboratory of Luminescent Materials and Devices, South China University of Technology, Guangzhou 510640, China

Prof. B. Z. Tang

AIE Institute, Guangzhou Development District, Huangpu, Guangzhou 510530, China

H. Liu, H. Bai and N. Yan equally contributed to this work.

Keywords: Optical engineering; cyanobacteria; aggregation-induced emission; algae factory 


\begin{abstract}
:
Human population is now faced with grand challenges such as global warming, food shortage and energy sustainability, which could be partially solved by massively increasing the growth and yield of photosynthetic organisms which capture the light energy to convert carbon dioxide and water into usable chemical energy. Cyanobacteria and eukaryotic microalgae are considered as attractive targets to be exploited by the algal factory because of their fast growth, low cost cultivation, less arable land and the diversity of high-value chemical substances produced. Many optical approaches have been introduced to increase the efficiency in artificial culturing systems, such as adding a luminescent layer that absorbs ultraviolet light and emits photosynthetic active radiation for cyanobacteria. In this work, we introduced luminogens with aggregationinduced emission characteristics (AIEgens) into the growth medium of a marine cyanobacteria. These hydrophobic AIEgens formed highly emissive luminogenic aggregates in the aqueous medium and dispersed around the cyanobacteria. Remarkedly, the number of cyanobacteria incubated in the medium with AIE aggregates was 5-fold more than the control group after 14-day culturing. The increased photosynthetic active radiation and the change of cyanobacteria protein expression in photosynthesis and metabolism might be the reason. Our study is the first using organic luminogenic aggregates as optical engineering inside the growth medium to dramatically increase the growth of cyanobacteria and demonstrated that AIEgens is promising technologies in the development of algal factories.
\end{abstract}




\section{Introduction}

Global warming, shortage of foods and lack of sustainable energy are the global grand challenges in the $21^{\text {st }}$ century. ${ }^{[1]}$ The massive usage of fossil fuels has generated large amount of carbon dioxide, one of the primary greenhouse gases. On the other hand, fossil fuels are classified as non-renewable resources which are being depleted much faster than the new ones are generated. Therefore, efficient utilization of clean and renewable energy is critically important to maintain sustainable development. Solar energy is the most abundant energy resource on earth. There are a variety of technologies that have been developed to take advantage of solar energy. For examples, semiconductor-based solar panels that convert the sun's light into usable electricity have been developed, while their conversion efficiency is still not high enough with high cost. $^{[2]}$ On the other hand, photosynthesis is a highly efficient light energy utilization method evolved naturally. ${ }^{[3]}$ Oxygenic photosynthetic organisms such as plants and algae harvest light energy to convert atmospheric carbon dioxide and water into oxygen and usable biomass such as carbohydrate, lipids and other bioactive metabolites. ${ }^{[4]}$ Although mass cultivation of the oxygenic photosynthetic organisms is a promise in solving these global challenges, the amount and productivity of oxygenic organisms are far below the demand for energy. ${ }^{[5]}$

Some startup algae and plant factories have been established in order to massively increase the amount and yield of photosynthetic species. ${ }^{[6]}$ Cyanobacteria (CB) can dominate marine environments and have been reef builders on Earth for more than three billion years. As most ancient oxygenic photosynthetic species, CB are a great promise in algae factory because they grow very fast and can be cultured without fresh-water and arable lands. ${ }^{[7]} \mathrm{CB}$ has some strong and broad absorption bands in the light spectrum due to the different pigments inside. ${ }^{[8]}$ Chlorophyll $\mathrm{a}$ and $\mathrm{b}$ are present in photosynthetic organisms (cyanobacteria, algae and plant) which have absorbance peaks in the blue and red regions (440 and $680 \mathrm{~nm}$ ). In addition to chlorophyll, there is principal light harvesting complex in the $\mathrm{CB}$ that is phycobilisome. ${ }^{[9]}$ The phycobilisome absorbs the green and yellow lights (500-650 $\mathrm{nm}$ range), which are inaccessible to chlorophyll. Therefore, their presence allows absorption and transfer of light energy to chlorophyll of the photosystem for photosynthesis. ${ }^{[10]}$ Although the future of algae factory is fascinating, the yield of cyanobacteria is not satisfactory, which is limited by various conditions such as light intensity and quality. ${ }^{[11]}$ 
Some efforts have been made to increase the growth rate and productivity of CB by optical engineering approaches, which basically increase the photosynthetic active radiation (PAR) and energy and thus the growth speed. ${ }^{[12]}$ About $30 \%$ increase in algae cell number was achieved by a luminescent material doped shifting layer, which shifted the ultra-violet (UV) light of sunlight into PAR. ${ }^{[13]}$ However, the overall setup of the shifting layer is labor intensive and not applicable in large scale operation. ${ }^{[13,14]}$ Furthermore, the fluorescent materials such as photoluminescent phosphor $\left(\mathrm{Ca}_{0.59} \mathrm{Sr}_{0.40} \mathrm{Eu}_{0.01} \mathrm{~S}\right)$ and Uvitex OB used in these earlier studies were either potentially toxic or inefficient light conversion. The simplest and efficient way to shift the UV light toward longer wavelength is to directly add the fluorescent materials into the algae growth medium. ${ }^{[15]}$ Organic fluorescent materials are more suitable because their biocompatibility is overall higher than inorganic materials. ${ }^{[16]}$ However, seawater system is very complicated due to their saline environment (normally $35 \%$ ) and the presence of various metallic and organic matters, which could induce the aggregation of organic fluorophores and lower the fluorescence efficiency due to the aggregationcaused quenching (ACQ). Their fluorescent intensity generally decreased or even quenched when they formed aggregates in aqueous medium. ${ }^{[17]}$ These ACQ fluorophores can absorb the short wavelength of sunlight but cannot efficiently emit the usable light for CB photosynthesis or growth. Therefore, traditional organic fluorescent materials cannot effectively work in the complicated seawater matrices, showing limited effects on promoting the growth and photosynthesis of marine cyanobacteria.

Aggregation-induced emission (AIE), on the contrary, is an opposite phenomenon, where the AIE fluorophores emit extensively in the aggregated state. ${ }^{[18]}$ Due to their good biocompatibility, high photostability and intense emission, many biological applications of AIE luminogens (AIEgens) have been explored, including biosensing, bioimaging and image-guided therapy. ${ }^{[19]}$ In the present study, two previously reported AIEgens (TPBA and APO) were used as the models to promote photosynthetic CB growth (Scheme 1). These hydrophobic AIEgens formed highly emissive luminogenic aggregates in the aqueous medium as well as around CB. The aggregates absorbed the UV and blue light and emitted efficient green fluorescence, serving as numerous and multidirectional nano light sources. With the addition of the AIE aggregates, we demonstrated that the growth rate of CB increased by 5-fold after 14-day culture as compared to the normal algal cultivation. We further conducted 
proteomic analysis and the result also suggested increased protein expression in pathways of photosynthesis and metabolism, which in turn supported the dramatically increased growth rate.

\section{Experiment Section}

Materials: All chemicals and reagents were commercially available and used as received without further purification. 2,6-Dichlorophenolindophenol (DCPIP), methyltert-butyl ether (MTBE), F/2 media, dithiothreitol (DTT), iodoacetamide (IAA) were purchased from Sigma. Cyanobacteria, Synechococcus bacillaris WH5701(CCMP1333) was obtained from Institute of Hydrobiology, Chinese Academy of Sciences. The AIEgens 3-diphenylamino-6-(2-pyridinyl)phenyldiphenylboron (TPBA) and 4-((2,2-difluoro-5-phenyl-2,3-dihydro-1,3,4,2-oxadiazaborol-3ylidene)methyl)-N,N-dimethylaniline (APO) were synthesized according to the previous procedure. ${ }^{[20]}$

Instrumentation: Absorption spectra were measured on a PerkinElmer UV/VIS Lambda 365 spectrophotometer. Steady-state photoluminescence (PL) spectra were measured on a Horiba FL-3000 fluorescence spectrophotometer. Particle size analysis was determined at room temperature using a Zetaplus Potential Analyzer (Brookhaven Instruments Corporation, USA). Laser confocal scanning microscope images were collected and analyzed on Zeiss laser scanning confocal microscope (LSM810). Treatment effects on the photophysiology of $S$. bacillaris were assessed with a fast repetition rate fluorometer (FRRf) in combination with a FastAct Laboratory system (FastOcean PTX), both from Chelsea Technologies Group Ltd. (West Molesey, UK).

Cyanobacteria imaging: S. bacillaris were concentrated to $10^{7}$ cells $/ \mathrm{mL}$ by centrifugation at $2200 \mathrm{~g}$, followed by incubation with TPBA or APO $(10 \mu \mathrm{M})$ for $1 \mathrm{~h}$. The cyanobacteria were imaged under a confocal microscope (Zeiss LSM 810 Laser Scanning Confocal Microscope), using proper excitation and emission filters: APO and TPBA, excitation $=405 \mathrm{~nm}$ and emission filter $=420-550 \mathrm{~nm}$; AIEgen sensitized cyanobacteria fluorescence, excitation $=405 \mathrm{~nm}$ and emission filter $=600-740 \mathrm{~nm}$; intrinsic cyanobacteria autofluorescence, excitation $=488 \mathrm{~nm}$ and emission filter $=$ $600-740 \mathrm{~nm}$. 
Cyanobacterial culture: Cyanobacteria was grown in $\mathrm{f} / 2$ media, ${ }^{[21]}$ under continuous illumination at a photon flux density of $30 \mu \mathrm{mol}$ of photons $\mathrm{m}^{-2} \mathrm{~s}^{-1}$, on a 16/8 light/dark cycle. Cell growth was monitored by using a traditional hemocytometer-based method under the microscope (Olympus cx31rtsf).

Impact of AIEgens on the growth of cyanobacteria: To investigate the capability of AIEgens in promoting the growth of cyanobacteria, S. bacillaris $\left(1.0 \times 10^{4}\right.$ cells $\left./ \mathrm{mL}\right)$ was exposed to TPBA or APO $(10 \mu \mathrm{M})$. At different time intervals, the cell number was counted by using the method mentioned above. The influence of UV light was evaluated by exposing the algae to UV light (wavelength: $315-400 \mathrm{~nm}$ ) for additional $1.5 \mathrm{~h}$ after the normal culture condition, which was similar to previous study. ${ }^{[22]}$ The cells were grown at two different $\mathrm{CO}_{2}$ levels with or without the bubbling aeration system of atmospheric $\mathrm{CO}_{2}$ level. Again, the cell number was counted at different time intervals.

Biomass analysis: S. bacillaris cells were first collected by centrifugation (2200 g, 5 $\mathrm{min})$, then washed with a $1 \%(\mathrm{w} / \mathrm{v}, \mathrm{g} / 100 \mathrm{~mL})$ aqueous $\mathrm{NaCl}$ solution, centrifuged again and freeze-dried. The dry biomass was analyzed immediately or stored at $-22{ }^{\circ} \mathrm{C}$ for up to 10-days prior to analysis.

Determination of lipid content: Lipid content was determined based on the previous study. ${ }^{[23]}$ In brief, for $200 \mathrm{~mL}$ of $S$. bacillaris, $1.5 \mathrm{~mL}$ of methanol was added and mixed rigorously (vertexing), followed by addition of $5 \mathrm{~mL} \mathrm{MTBE}$, and the mixture was incubated for $1 \mathrm{~h}$ at room temperature. Water $(1.25 \mathrm{~mL})$ was added to the mixture and allowed to stand at room temperature for $10 \mathrm{~min}$ to develop phase separation. The upper organic phase was collected after centrifugation at $1000 \times \mathrm{g}$ for $10 \mathrm{~min}$. The lower phase was re-extracted with a new addition of $2 \mathrm{~mL} \mathrm{MTBE/methanol/water} \mathrm{(10/3/2.5,}$ $\mathrm{v} / \mathrm{v} / \mathrm{v}$ ) to achieve complete lipid recovery. The organic phase containing the lipid extract was vacuum dried, and the dried vials were weighed, and lipid content was measured gravimetrically. Lipid production $\left(\mathrm{g} \mathrm{L}^{-1}\right)$ was determined only at the end of the culture period.

$\boldsymbol{F}_{\mathrm{v}} / \boldsymbol{F}_{\mathbf{m}}$ detection: Variable fluorescence, $F_{\mathrm{v}}$, was calculated by subtracting maximal, $F_{\mathrm{m}}$, with initial fluorescence, $F_{0}$. The ratio $F_{\mathrm{v}} / F_{\mathrm{m}}$ was a measure of the quantum efficiency if all PSII centres were open, i.e., maximum photosynthetic efficiency, and was highly correlated with the quantum yield of net photosynthesis. ${ }^{[24]}$ Treatment 
effects on the photophysiology of $S$. bacillaris were assessed with a fast repetition rate fluorometer (FRRf) in combination with a FastAct Laboratory system (FastOcean PTX), both from Chelsea Technologies Group Ltd. (West Molesey, UK). Excitation wavelength of the fluorometer's LED was $450 \mathrm{~nm}$ with an automated adjustment of the light intensity to $0.66-1.2 \times 10^{22} \mu \mathrm{mol}$ photons $\mathrm{m}^{-2} \mathrm{~s}^{-1}$. A single turnover mode with 100 flashlets saturation phases on a $2 \mu$ s pitch and 40 flashlets relaxation phases on a $40 \mu$ s pitch was used to increasingly saturate the PSII. Iterative algorithms for the induction $^{[25]}$ and relaxation phases ${ }^{[26]}$ were applied to estimate the minimum Chl a fluorescence $\left(F_{0}\right)$ and maximum $\mathrm{Chl}$ a fluorescence $\left(F_{\mathrm{m}}\right)$. The apparent maximum quantum yield of photosynthesis of PSII $\left(F_{\mathrm{v}} / F_{\mathrm{m}}\right)$ could then be calculated.

Proteomics Analysis: The control and AIEgens treated cyanobacteria cultured after 14 days were collected. Then, the proteins were extracted from cells and tryptic peptides were prepared for standard shotgun proteomics using LC-MS/MS analysis. The raw data acquired by the mass spectrometer was processed and searched against the Synechococcus sp. WH 5701 protein sequence database. Label-free quantification was applied for determining the relative amount of proteins in both control and AIEgens treated groups based on spectral counting. To better understand the significantly regulated proteins, protein networks were studied. Details regarding LC-MS/MS analysis method, proteomics data analysis and bioinformatics analysis were included in the supplementary information.

\section{Results and Discussion:}

\section{Absorbance and fluorescence coupling of AIEgen and Cyanobacteria:}

Cyanobacteria was mainly cultured under two types of light sources: natural sunlight and artificial white LED light. Therefore, the absorption spectrum of cyanobacteria $(\mathrm{CB})$ and the emission profiles of the light sources were first measured to evaluate the energy matching degree between $\mathrm{CB}$ and the light sources. As shown in Figure 1A, CB exhibited broad absorption bands in the blue region and red region (peaks around 450nm, 640nm and $680 \mathrm{~nm}$ ) correlated to the chlorophyll a and b, and green region (peaks around 500nm) correlated to the phycoerythrin. However, there were obvious mismatches between the cyanobacteria absorption and the two light sources. The strong UV light (300-400 nm) in sunlight was not absorbed by the CB for 
photosynthesis. Similarly, the LED light in the region of $450-550 \mathrm{~nm}$ did not overlap with the $\mathrm{CB}$ absorption. These data indicated that the $\mathrm{CB}$ could not efficiently capture and covert the sunlight or artificial light for photosynthesis and growth. These measurements strongly suggested that new material is required to convert the nonutilized light energy to usable light to be captured by CB.

The AIEgens with high quantum yield and matched absorption and emission spectrum were the promising candidates. The TPBA and APO were employed in the following investigations, which owned greenish-blue fluorescence emission with high quantum yield (88.4\% and $25 \%$ respectively). ${ }^{[20]}$ The particle size and photophysical property of the AIEgens were measured in seawater. Dynamic light scattering (DLS) results showed that the average particle sizes of TPBA were around $260 \mathrm{~nm}$ and APO were around $150 \mathrm{~nm}$ (Figure S1). The UV-vis absorption spectra of TPBA and APO showed that they both exhibited strong absorptivity in the UV and blue regions with the peaks around $420 \mathrm{~nm}$ and $380 \mathrm{~nm}$, respectively (Figure 1B). The photoluminescence (PL) spectra showed that the TPBA emitted intense fluorescence in $450-550 \mathrm{~nm}$ (Figure 1C). After adding the algae in the seawater, the green fluorescence of TPBA decreased, while a new red fluorescent peak around $680 \mathrm{~nm}$ increased. The excitation of chlorophyll could cause charge separation and photochemical reaction, and the separated charges could recombine with bright fluorescence emission at around $680 \mathrm{~nm}$ with high intensity of light. ${ }^{[27]}$ Thus, the enhanced red fluorescence was from the chlorophyll in the CB. Similarly, the emission of APO covered $500 \mathrm{~nm}$ to $650 \mathrm{~nm}$ and exhibited a similar trend with the TPBA after adding CB (Figure 1D). Moreover, they both exhibited the energy transfer function under the excitation of $350 \mathrm{~nm}$ and $450 \mathrm{~nm}$ (Figure S2), suggesting that the reabsorption of cyanobacteria from luminescence of AIEgens was possible under a broad spectrum of UV and blue lights. Subsequently, the roles of TPBA and APO in the CB photosynthesis were tested with the common probe DCPIP. During the light-dependent reaction, DCPIP could capture and react with the produced electron from pigment chlorophyll, leading to the decreased absorbance around $600 \mathrm{~nm}$, which could reflect the electron generation and transportation in photosynthesis. As shown in Figure S3, the absorbance of DCPIP in CB mixed AIEgen groups decreased much faster than the control group under light irradiation, which demonstrated that the AIEgens could transfer the light energy to $\mathrm{CB}$ and promoted the light-dependent reaction. 


\section{Confocal imaging of cyanobacteria with AIEgens:}

CLSM was used to investigate the spatial distribution and interaction between the AIE aggregates and the CB. No fluorescent signal was detected for the $\mathrm{CB}$ incubated in the growth medium under excitation of $405 \mathrm{~nm}$ (Figure 2C1 and 2C2). On the other hand, when the AIEgen was added to the $\mathrm{CB}$ growth medium, the fluorescence of AIEgens (green) and chlorophyll (red) were detected under excitation of $405 \mathrm{~nm}$ (Figure 2A1, A2, B1 and B2), while the red fluorescence of chlorophyll in all groups was observed under laser excitation of $488 \mathrm{~nm}$ (Figure 2A4, B4 and C4). By closer examining the merged images, we found that the two FL signals were spatially separated, suggesting that the AIE aggregates surrounded the CB (Figure $2 \mathrm{A3}$ and B3). To further investigate the long-lasting stability during the algal growth, the $\mathrm{CB}$ mixed with TPBA and APO were imaged after 14 days of culture. The results showed that the formed AIE aggregates dispersed around the CB in the growth medium (Figure S4). Thus, the emission of AIE aggregates could luminate the CB under the light excitation. The light radiation by AIEgens could be effectively reabsorbed by $\mathrm{CB}$ and the converted energy could be used for CB photosynthesis.

\section{Growth of cyanobacteria}

We first studied the cytotoxicity of AIE materials to the cyanobacteria. There was negligible cytotoxicity of TPBA and APO toward cyanobacteria up to $20 \mu \mathrm{M}$ (Figure S5). Then, the growth of cyanobacteria was monitored under the condition of $16 \mathrm{~h}$ white light LED illumination and $8 \mathrm{~h}$ dark cycles. It was obvious that the cell density dramatically increased under TPBA and APO treatment (Figure 3A). The differences occurred on Day 3, and the maximum fold change was about five in the two AIEs treated groups compared to the control after 14-day culture. Apart from the cell density, the biomass and lipid production of cyanobacteria were also measured. Clearly, both the biomass and lipid production increased in both TPBA treated and APO treated group, consistent with the cell density measurement (Figure 3B and 3C). Thus, with AIE aggregates the light could be much more efficiently utilized by the cyanobacteria, resulting in higher photosynthetic efficiency and growth rate. Our results demonstrated the great potential of AIE aggregates as a boosting agent, promoting the growth and 
photosynthesis activity of cyanobacteria (or even other photosynthetic species), eventually increasing the production of biofuel. Besides the light source, the availability of $\mathrm{CO}_{2}$ could be another parameter that limited the growth and photosynthetic activity of cyanobacteria. Accordingly, cyanobacteria were cultured with continuous supply of the $\mathrm{CO}_{2}(0.035 \%)$ and compared with that cultured under normal condition. The result showed that the cell concentration of TPBA or APO treated cyanobacteria with constant supply of $\mathrm{CO}_{2}$ increased about $50 \%$ as compared to that without supply of $\mathrm{CO}_{2}$ (Figure 3D). Under this condition, the cell concentration reached about 9 times more than the control group cultured without adding AIEgens and $\mathrm{CO}_{2}$ supply, which is promising in culturing cyanobacteria and other photosynthetic organisms.

As natural sunlight is the most available light source for photosynthesis, the growth of cyanobacteria under natural sunlight was also studied. The cell concentration increased after the incubation with TPBA or APO under $12 \mathrm{~h}$ sunlight and dark (Figure 3E). Considering that AIE aggregates absorbs UV light, which is harmful to cyanobacteria, the protection effect of AIE aggregates was studied. Under UV light irradiation $(1.5 \mathrm{~h})$ after the white light incubation $(16 \mathrm{~h})$, cyanobacteria were unable to grow due to UV light, but were able to grow with incubation of TPBA or APO (Figure 3F). Furthermore, we found that the protection effect was associated with the concentration of AIE aggregates.

\section{Adaptation of cyanobacteria in the AIE aggregates environment}

The spatial and spectrum change of light distribution caused by AIE aggregates created unique environment for cyanobacteria to grow. Earlier study indicated that the protein expression changed in response to light quality. ${ }^{[28]} \mathrm{We}$ then examined the photosynthesis and protein responses to AIE aggregates. We measured the maximum photosynthetic quantum yield $\left(F_{\mathrm{v}} / F_{\mathrm{m}}\right)$ and efficiency of protein machinery of light reaction in cyanobacteria. The results showed that the quantum yield in TPBA and APO treatments was much higher than the control during the cell culture (Figure 4A), reaching earlier to around 0.8 than the control group. This value was maintained afterwards as the optimum state of light reaction. Thus, the protein machinery changed to its optimal state under this specific environment. Detailed proteomic changes were studied after 14-day of incubation with AIE aggregates. There were 188 common 
proteins found in APO treatment and control, 187 common proteins in TPBA and control, and 166 common proteins in APO treatment, TPBA treatment and control (Figure S6). Next, differences in protein expression were identified with fold change higher or lower than \pm 1.5 and $p$-values from a t-test $<0.05$ (Figure S7).

To get a further insight of the influence of AIE aggregates on protein expression of cyanobacteria, proteins that are significantly up-regulated or down-regulated in both APO and TPBA treatments were plotted in a heat map with color indicating the fold change (Figure 4B). There were 21 proteins up-regulated and 18 proteins downregulated characterized respectively with identical name and function. Among the upregulated proteins, those related to photosynthesis and metabolism were marked with a red star. These proteins were then classified in different groups including phycobilisome, photosystem II, photosystem I, ATP synthesis, carbon fixation and metabolism (Figure 4C). In these groups, proteins were identified including phycocyanin, phycobilisome rod-core linker, Photosystem II CP47 reaction center protein, Photosystem II D2 protein, Photosystem I reaction center, NAD(P)H-quinone oxidoreductase, thioredoxin, phosphoribulokinase, ATP-synthase, pyruvate kinase and so on. Many proteins are in the core of the protein machinery such as light reaction, ATP synthesis and carbon fixation. ${ }^{[29]}$ Detailed differentially expressed proteins between APO-treated group and normal as well as proteins between TPBA-treated group and normal were identified and studied respectively (Figure S8). These results indicated that the unique environment created by AIE aggregates induced the change of protein expressions of cyanobacteria, which supported the dramatically enhanced photosynthesis and growth rate.

\section{Conclusion:}

Algae may provide sustainable foods and biofuels, as well as reduce the atmospheric carbon dioxide. Cyanobacteria is a promising candidate due to their widespread distribution and importance in the ocean environment. In this study, we introduced highly emissive organic luminogenic AIE aggregates (TPBA and APO) inside the growth medium and demonstrated the dramatic increase of photosynthesis and growth of cyanobacteria. The APO and TPBA absorbed the UV and blue light from the light source, which could cause harmful effect to cyanobacteria. Further, the highly 
efficient fluorescence of AIE aggregates in the range of 450-600 nm was reabsorbed by cyanobacteria for photosynthesis. The increased photosynthetic active radiation by both spectra shifting and spatial surroundings of APO and TPBA aggregates dramatically enhanced the growth rate of cyanobacteria, leading to as much as five times faster than control. At the meantime, cyanobacteria adapted to the unique environment created by APO and TPBA aggregates, with up-regulated protein expression in light harvesting, light reaction, ATP synthesis, carbon fixation, which correlates to the enhanced photosynthesis and growth. Further studies are required to explore the effects of size, absorbance and emission spectrum of AIE aggregates. This optical engineering by utilizing organic luminogenic aggregates provides an exciting and promising way to develop algae and plant factory and solving the global challenges.

\section{Conflicts of interest}

There are no conflicts to declare

\section{Acknowledgements}

This work is supported by Research Grants Council of Hong Kong (N_HKUST604/14 and C6009-17G), the Innovation and Technology Commission (ITC-CNERC14SC01), and the National Key Research and Development program of China (2018YFE0190200), the Science and Technology Plan of Shenzhen (JCYJ20160229205601482 and JCYJ20180507183832744). 


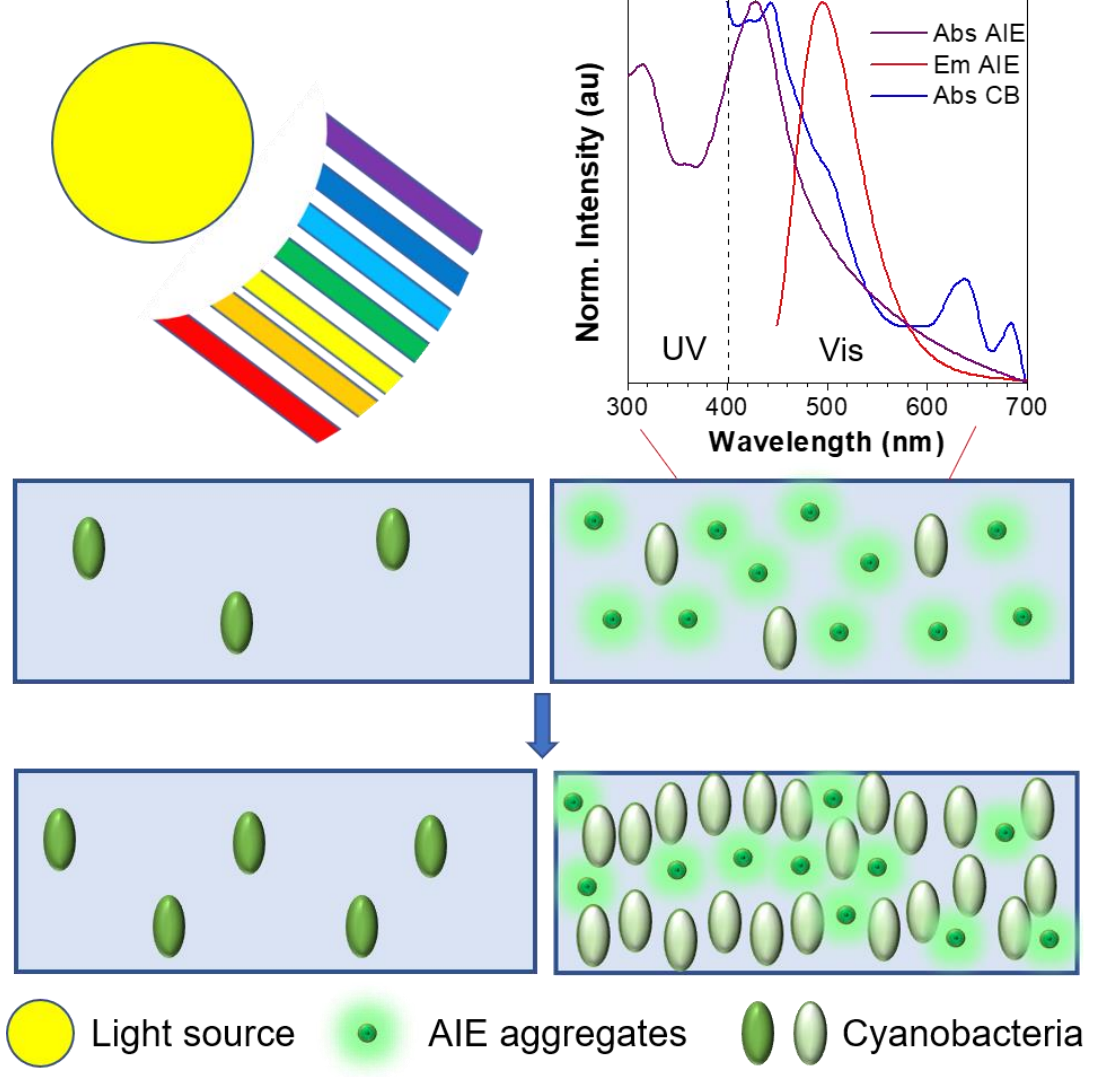

Scheme 1. Schematic illustration of luminogenic AIE aggregates for dramatically enhanced photosynthesis and growth of cyanobacteria. UV: ultraviolet; Vis: visible light; Abs: absorption; Em: emission; CB: cyanobacteria 

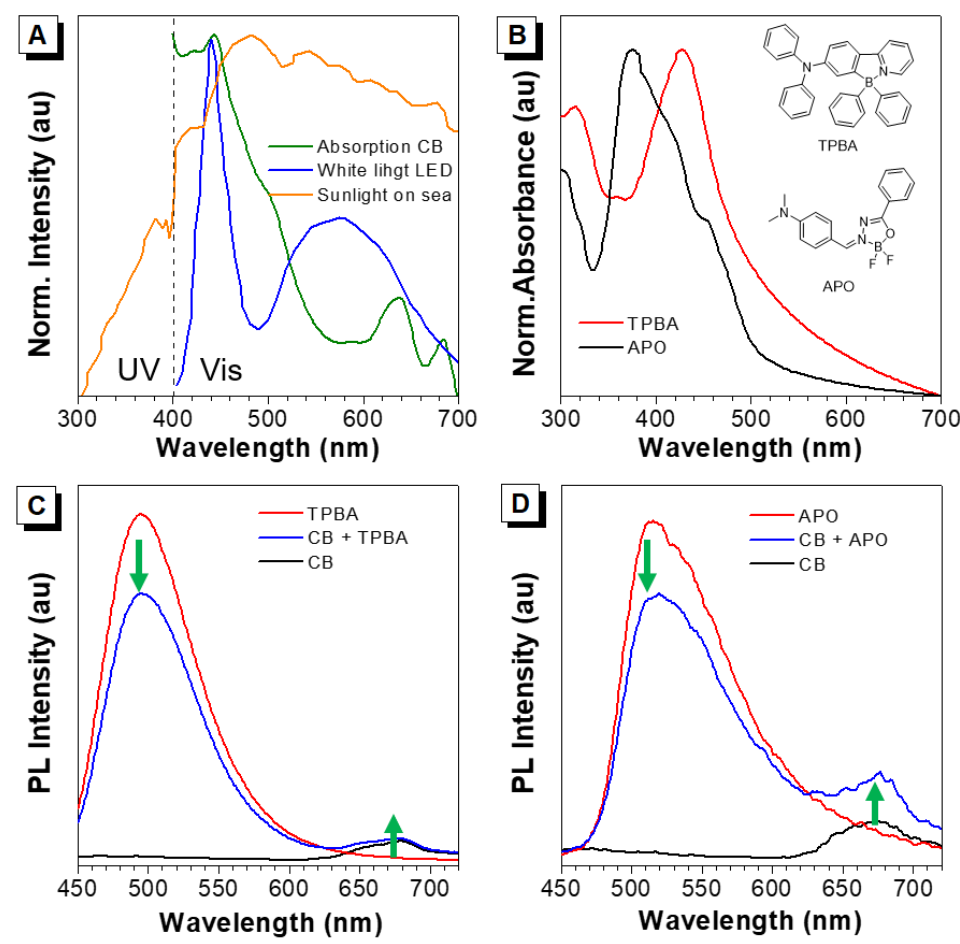

Figure 1. Absorbance and photoluminescence (PL) of APO, TPBA, cyanobacteria (CB) and their combination. (A) The absorption of cyanobacteria in seawater and the light spectrum of sunlight on sea level and commercially used cool light LED light for plant growth; (B) the absorbance of TPBA and APO in seawater containing 0.1\% DMSO. (C) the PL spectrum of TPBA, cyanobacteria and cyanobacteria incubated with TPBA under the excitation of $400 \mathrm{~nm}$; (D) the PL spectrum of APO and cyanobacteria incubated with APO under the excitation of $400 \mathrm{~nm}$. APO and TPBA were used as $10^{-}$ ${ }^{5} \mathrm{M}$, the concentration of cyanobacteria is $10^{6}$ cells $/ \mathrm{mL}$. 


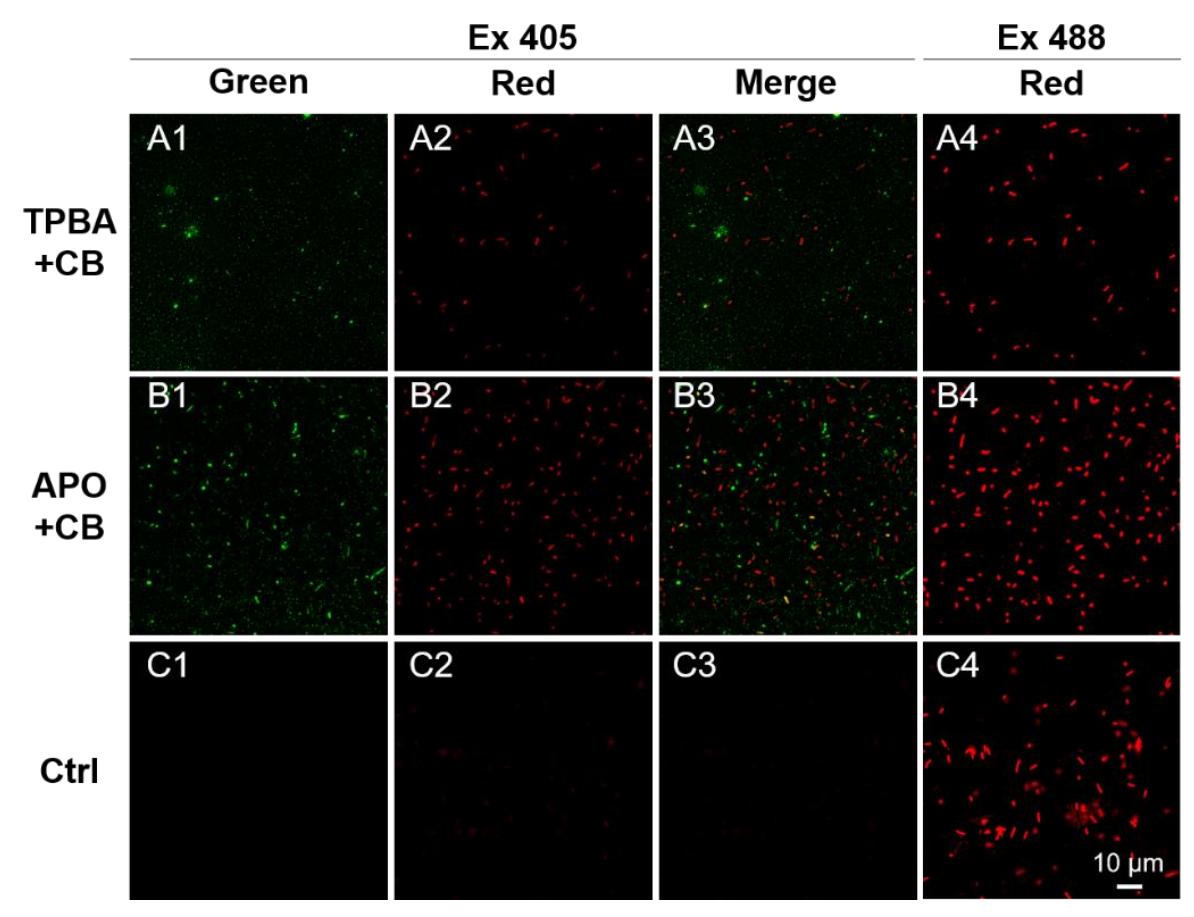

Figure 2. Confocal images of Cyanobacteria (C) and cyanobacteria stained with TPBA (A) and APO (B). Green signal (A1, B1, C1) and red signal (A2, B2, C2) was taken under excitation of $405 \mathrm{~nm}$, which represents the fluorescence of APO or TPBA and the cyanobacteria. Merged images (A3, B3, C3) were merged by green and red signal. Another red signal (A4, B4, C4) was taken under excitation of $488 \mathrm{~nm}$, which represents autofluorescence signal excited directly by laser. APO and TPBA were used as $10^{-5} \mathrm{M}$, the concentration of cyanobacteria is $10^{7}$ cells $/ \mathrm{mL}$. 

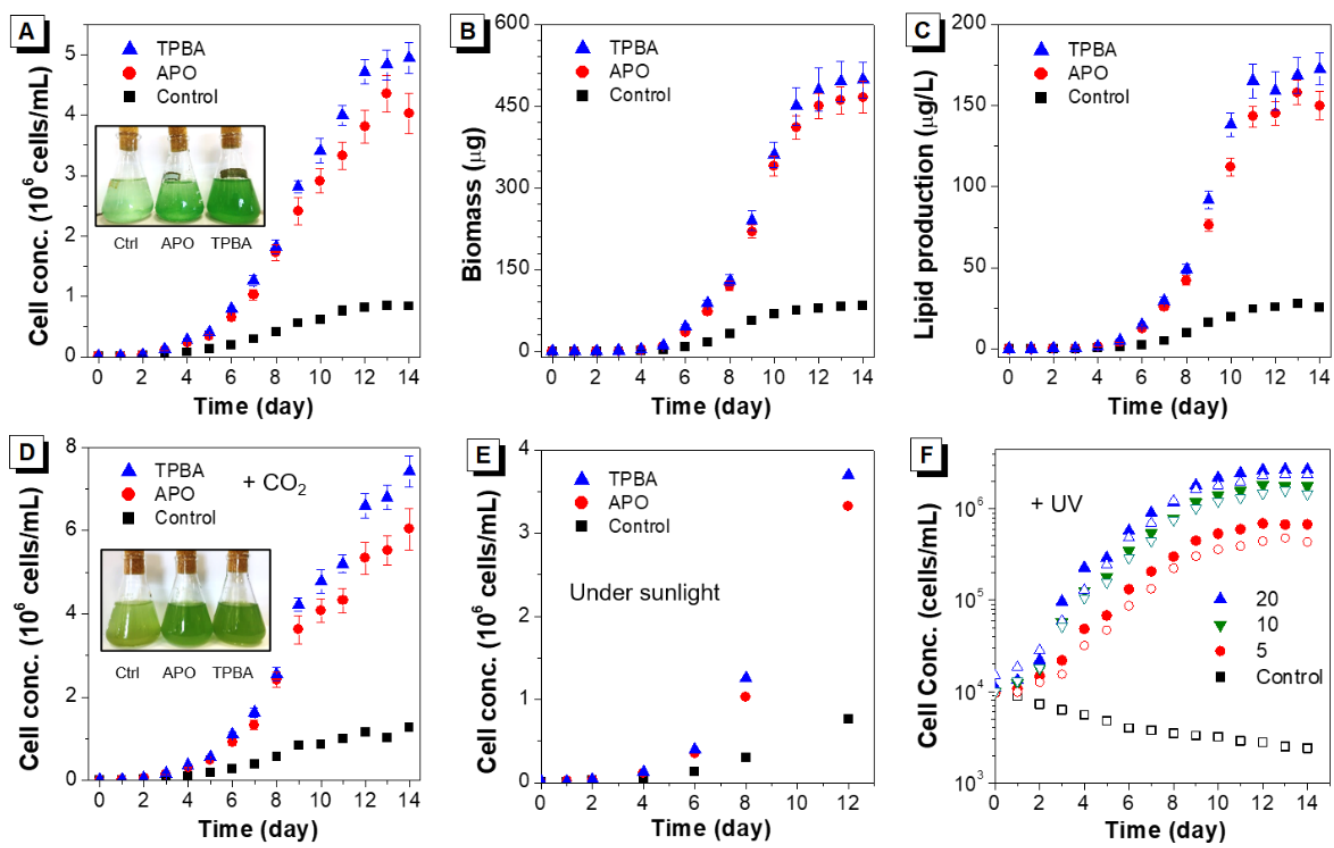

Figure 3. Growth of cyanobacteria with or without AIE aggregates. (A) the cell concentration, (B) the biomass of dry weight and (C) the lipid production of cyanobacteria incubated with APO or TPBA under $16 \mathrm{~h}$ white light, $8 \mathrm{~h}$ dark cycle for a growth period of 14 days;(D) the growth curve of cyanobacteria and cyanobacteria with APO or TPBA under $16 \mathrm{~h}$ white light irradiation and $8 \mathrm{~h}$ dark under supply of carbon dioxide; (E) the growth of cyanobacteria and cyanobacteria incubated with TPBA or APO under $12 \mathrm{~h}$ sunlight near the sea for 12 days; (F)the growth curve of cyanobacteria incubated with different concentrations of TPBA (solid) or APO (hallow) under $16 \mathrm{~h}$ white light, $1.5 \mathrm{~h} \mathrm{UV}$ and 6.5 dark cycle for 14 days. Concentration of APO or TPBA is $10^{-5} \mathrm{M}$. Cyanobacteria were seeded as $10^{4}$ cells $/ \mathrm{mL}$. Insert are photographs of cyanobacteria grown after 14 days. 

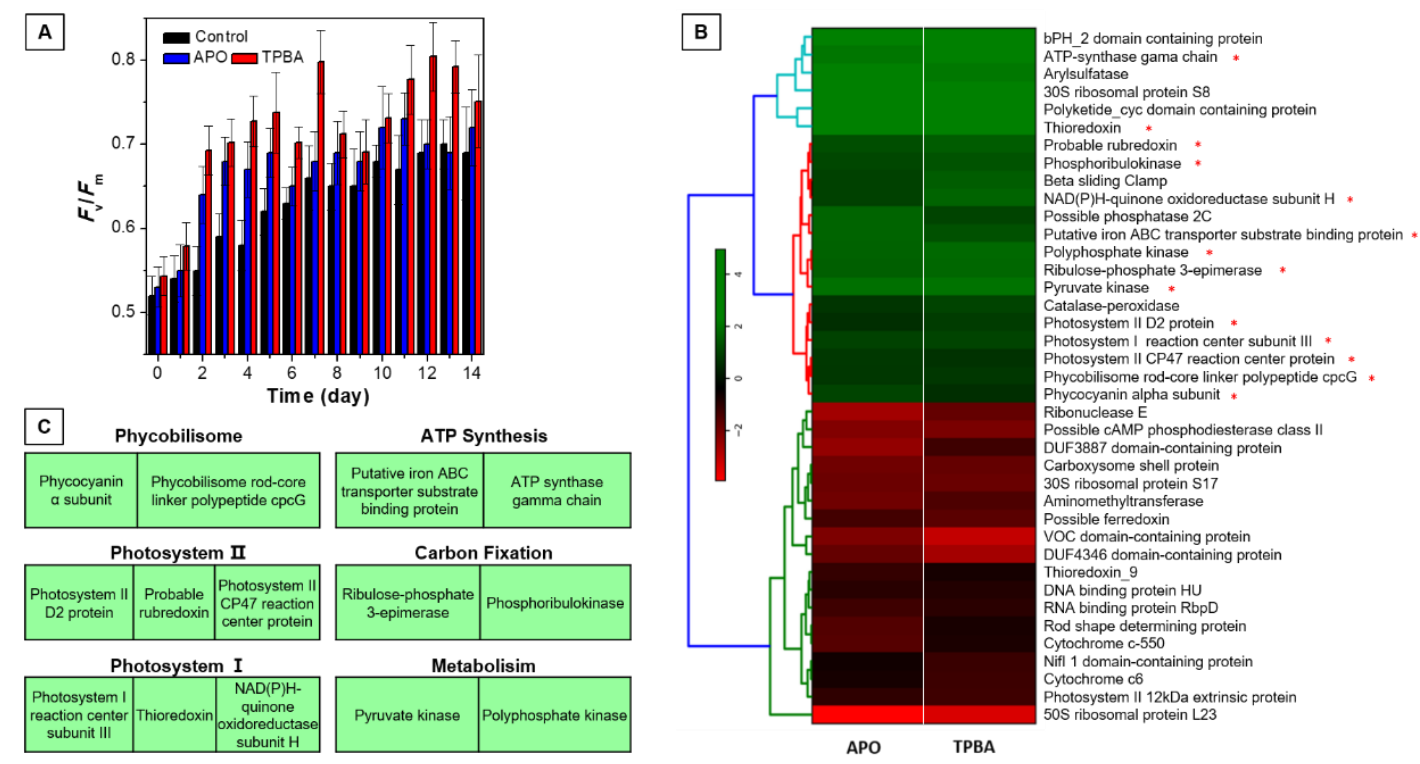

Figure 4. The change of cyanobacteria protein machinery after incubation with TPBA or APO. (A) the maximum quantum yield $\mathrm{Fv} / \mathrm{Fm}$ of cyanobacteria under normal condition and that incubated with $10^{-5} \mathrm{M}$ TPBA or APO for 14 days; (B) Heat map of the differentially expressed proteins in TPBA-treated WH5701 and APO-treated WH5701 compared to the normal WH5701. Proteins are defined if all the fold changes are above or below 1, and p-values are $<0.05$. (C) Schematic diagram of possible increases in groups of photosynthesis pathway in WH5701 after the treatment of TPBA or APO. 


\section{Reference:}

[1] J. P. Dorian, H. T. Franssen, D. R. Simbeck, Energ Policy 2006, 34, 1984; M. W. Rosegrant, S. A. Cline, Science 2003, 302, 1917; K. E. Trenberth, A. G. Dai, G. van der Schrier, P. D. Jones, J. Barichivich, K. R. Briffa, J. Sheffield, Nat. Clim. Change 2014, 4, 17.

[2] E. Kabir, P. Kumar, S. Kumar, A. A. Adelodun, K. H. Kim, Renew. Sust. Energ. Rev. 2018, 82,894 .

[3] J. Barber, Chem. Soc. Rev. 2009, 38, 185.

[4] D. R. Ort, S. S. Merchant, J. Alric, A. Barkan, R. E. Blankenship, R. Bock, R. Croce, M. R. Hanson, J. M. Hibberd, S. P. Long, T. A. Moore, J. Moroney, K. K. Niyogi, M. A. J. Parry, P. P. Peralta-Yahya, R. C. Prince, K. E. Redding, M. H. Spalding, K. J. van Wijk, W. F. J. Vermaas, S. von Caemmerer, A. P. M. Weber, T. O. Yeates, J. S. Yuan, X. G. Zhu, P. Natl. Acad. Sci. 2015, 112, 8529.

[5] S. P. Long, A. Marshall-Colon, X. G. Zhu, Cell 2015, 161, 56.

[6] B. J. Ding, P. Hofvander, H. L. Wang, T. P. Durrett, S. Stymne, C. Lofstedt, Nat. Commun. 2014, 5, 3353.

[7] N. E. Nozzi, J. W. Oliver, S. Atsumi, Frontiers in bioengineering and biotechnology 2013, 1, 7; S. A. Angermayr, K. J. Hellingwerf, P. Lindblad, M. J. T. de Mattos, Curr. Opin. Biotech. 2009, 20, 257.

[8] C. S. Ting, G. Rocap, J. King, S. W. Chisholm, Trends Microbiol. 2002, 10, 134.

[9] A. R. Grossman, D. Bhaya, Q. F. He, J. Biol. Chem. 2001, 276, 11449.

[10] J. Nogales, S. Gudmundsson, E. M. Knight, B. O. Palsson, I. Thiele, P. Natl. Acad. Sci. 2012, 109, 2678.

[11] P. G. Stephenson, C. M. Moore, M. J. Terry, M. V. Zubkov, T. S. Bibby, Trends Biotechnol. 2011, 29, 615.

[12] M. D. Ooms, C. T. Dinh, E. H. Sargent, D. Sinton, Nat. Commun. 2016, 7, 12699.

[13] L. Wondraczek, M. Batentschuk, M. A. Schmidt, R. Borchardt, S. Scheiner, B. Seemann, P. Schweizer, C. J. Brabec, Nat. Commun. 2013, 4, 2047.

[14] H. D. Amrei, B. Nasernejad, R. Ranjbar, S. Rastegar, J. Appl. Phycol. 2014, 26, 1493; H. D. Amrei, R. Ranjbar, S. Rastegar, B. Nasernejad, A. Nejadebrahim, J. Appl. Phycol. 2015, 27, 67.

[15] A. Prokop, M. F. Quinn, M. Fekri, M. Murad, S. A. Ahmed, Biotechnology and bioengineering 1984, 26, 1313.

[16] X. Y. Zhang, S. Q. Wang, L. X. Xu, L. Feng, Y. Ji, L. Tao, S. X. Li, Y. Wei, Nanoscale 2012, 4, 5581.

[17] R. F. Chen, J. R. Knutson, Anal. Biochem. 1988, 172, 61; T. Luo, T. Zhou, Y. H. Zhao, L. W. Liu, J. L. Qu, J. Mater. Chem. B 2018, 6, 1912.

[18] J. Mei, N. L. C. Leung, R. T. K. Kwok, J. W. Y. Lam, B. Z. Tang, Chem. Rev. 2015, $115,11718$.

[19] R. T. K. Kwok, C. W. T. Leung, J. W. Y. Lam, B. Z. Tang, Chem. Soc. Rev. 2015, 44, 4228; Z. Zheng, T. F. Zhang, H. X. Liu, Y. C. Chen, R. T. K. Kwok, C. Ma, P. F. Zhang, H. H. Y. Sung, I. D. Williams, J. W. Y. Lam, K. S. Wong, B. Z. Tang, Acs Nano 2018, $12,8145$. [20] D. F. Dang, H. X. Liu, J. G. Wang, M. Chen, Y. Liu, H. H. Y. Sung, I. D. Williams, R. T. K. Kwok, J. W. Y. Lam, B. Z. Tang, Chem. Mater. 2018, 30, 7892; J. S. Ni, H. X. Liu, J. K. Liu, M. J. Jiang, Z. Zhao, Y. C. Chen, R. T. K. Kwok, J. W. Y. Lam, Q. Peng, B. Tang, Mater. Chem. Front. 2018, 2, 1498.

[21] R. R. Guillard, in Culture of marine invertebrate animals, Springer, 1975, 29.

[22] U. Bickmeyer, S. Thoms, F. Koch, L. P. Mukagatare, R. Silalahi, F. J. Sartoris, PLoS One 2019, 14.

[23] V. Matyash, G. Liebisch, T. V. Kurzchalia, A. Shevchenko, D. Schwudke, J. Lipid Res. 2008, 49, 1137.

[24] K. Maxwell, G. N. Johnson, J. Exp. Bot. 2000, 51, 659.

[25] Z. S. Kolber, O. Prášil, P. G. Falkowski, Biochimica et Biophysica Acta (BBA)Bioenergetics 1998, 1367, 88. 
[26] K. Oxborough, C. M. Moore, D. J. Suggett, T. Lawson, H. G. Chan, R. J. Geider, Limnol. Oceanogr. Methods 2012, 10, 142.

[27] N. R. Baker, Annu. Rev. Plant. Biol. 2008, 59, 89.

[28] C. W. Mullineaux, Front. Plant Sci. 2014, 5, 7.

[29] N. Nelson, A. Ben-Shem, Nat. Rev. Mol. Cell Bio. 2004, 5, 971; T. Ogawa, H. Mi, Photosynth. Res. 2007, 93, 69; Y. Munekaga, M. Hashimoto, C. Miyaka, K. I. Tomizawa, T. Endo, M. Tasaka, T. Shikanai, Nature 2004, 429, 579. 